\section{Veinte años de cirugía reparadora de la válvula mitral}

\author{
RICARDO ZALAQUETT S., GONZALO LATORRE S.a, \\ JOSEFINA ALMEIDA Z. ${ }^{\text {, }}$, ALDO MENESES O. ${ }^{\mathrm{a}}$, \\ CECILIA MUÑOZ C., SAMUEL CÓRDOVA A.
}

\section{Twenty years of mitral valve repair. An experience in 322 patients}

Background: Mitral valve repair is the preferred procedure for the surgical treatment of mitral valve insufficiency (MI), procedure that we initiated 20 years ago. Aim: To assess our experience and long-term results of mitral valve repair. Patients and Methods: The database of the cardiology department was reviewed for the period between December 1991 and December 2012. A total of 322 patients aged 18 to 89 years (62\% males) undergoing mitral valve repair were identified. Long-term survival information was obtained consulting death certificate records of the Chilean Identification Service. The latest echocardiogram available was analyzed. Results: MI was degenerative in 144 patients (45\%) and ischemic in 104 (32\%). A prosthetic ring was used in all ischemic and in $92 \%$ of non-ischemic MI. Operative mortality was 7.5\%, 13\% in ischemic and $4.4 \%$ in non-ischemic $M I(p<0.01)$. Overall long-term survival was 14.1 years; 9.3 and 16 years for ischemic and non-ischemic MI, respectively $(p<0.001)$. Survival at $5,10,15$ and 20 years was 79, 63, 54 and 42\%, respectively. For degenerative MI survival at 5 and 10 years was 90 and $76 \%$ and for ischemic MI, it was 64 and $44 \%$, respectively $(p<0.001)$. On a multivariate analysis the main predictors of late mortality were age, associated valvular disease and ischemic etiology. Echocardiographic follow-up was available for 223 patients; MI was absent in 53\% and was mild in 29\%. Conclusions: In a 20 years follow-up, mitral valve repair for $M I$ had excellent long-term survival and echocardiographic results. The most common etiologies of MI were degenerative and ischemic diseases. The latter had a worst prognosis. The main predictors of long term mortality were age, associated valvular disease and ischemic etiology.

(Rev Med Chile 2014; 142: 1089-1098)

Key words: Mitral valve; Mitral valve annuloplasty; Mitral valve insufficiency; Thoracic surgery.

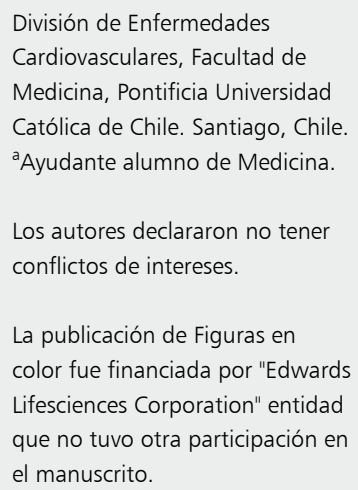

Recibido el 26 de abril de 2014, aceptado el 25 de agosto de 2014.

Correspondencia a:

Dr. Ricardo Zalaquett $\mathrm{S}$. División de Enfermedades Cardiovasculares, Pontificia Universidad Católica de Chile. Marcoleta 367, piso 8

Teléfono: 354 3231;

Fax: 6390108.

rzalaque@med.puc.cl
S i bien la importancia de la conservación del aparato valvular mitral a través de la cirugía reparadora de la insuficiencia mitral (IM) se reconoce desde la década de 1970, el camino para que esto fuera universalmente aceptado fue $\operatorname{largo}^{1,2}$.

En 1983 Carpentier definió la "Triada de Carpentier” -dada por la etiología, la lesión resultante y la disfunción secundaria a la lesión-introdujo una clasificación anatómica y funcional de la IM, describió los procedimientos quirúrgicos para la reparación de la válvula mitral $(\mathrm{VM})$ y presentó sus resultados alejados ${ }^{3}$. A partir de esta publicación, y con la introducción de la ecocardiografía transesofágica, la reparación mitral empezó a ser aceptada por algunos cirujanos norteamericanos, 
Reparación valvular mitral - R. Zalaquett et al

Tabla 1. Distribución por sexo y edad según etiología de la insuficiencia mitral

\begin{tabular}{|lccccccc|}
\hline Características & Degenerativa & Isquémica & Infecciosa & Reumática & Otras & Total & p \\
$\begin{array}{l}\text { Edad promedio } \\
\text { (IC 95\%) }\end{array}$ & 60 & 66 & 48 & 48 & 58 & 60 & 0,49 \\
Sexo (n y \%) & $(57,5-62,4)$ & $(64,6-67,9)$ & $(42,5-53,3)$ & $(40,5-55,4)$ & $49,4-66,6)$ & $(58,2-61,5)$ & \\
$\quad$ Masculino & $91(63,2)$ & $67(64,4)$ & $20(55,6)$ & $6(33,3)$ & $14(70,0)$ & $198(61,5)$ & $<0,001$ \\
$\quad$ Femenino & $53(36,8)$ & $37(35,6)$ & $16(44,4)$ & $12(67,7)$ & $12(30,0)$ & $124(38,5)$ \\
Total & $144(44,7)$ & $104(32,3)$ & $36(11,2)$ & $18(5,6)$ & $20(6,2)$ & $322(100)$ \\
\hline
\end{tabular}

que a fines de la década 1980-89 comenzaron a presentar sus resultados, llevando a que finalmente la reparación de la VM fuera universalmente aceptada como el procedimiento de elección para la corrección de la $\mathrm{IM}^{4-7}$.

En 1991 iniciamos nuestra experiencia en cirugía reparadora de la IM con control ecocardiográfico transesofágico intraoperatorio en todos los casos. En 1994 presentamos resultados iniciales y en 1996 lo hicimos con especial énfasis en la evaluación ecocardiográfica intraoperatoria ${ }^{8,9}$. En 1999 pudimos presentar resultados alejados globales, en 2003 lo hicimos exclusivamente para la IM degenerativa y en 2004 para la endocarditis infecciosa ${ }^{10-12}$. En 2005 presentamos nuestros "Resultados alejados de la reparación versus reemplazo en insuficiencia mitral degenerativa", confirmando la superioridad de la reparación por sobre el reemplazo mitral ${ }^{13}$. Finalmente, en 2011 comunicamos nuestros resultados en 100 pacientes con anuloplastía mitral por IM isquémica ${ }^{14}$.

En esta oportunidad comunicamos nuestra experiencia en 20 años transcurridos desde que iniciamos la cirugía reparadora de la IM.

\section{Pacientes y Método}

\section{Pacientes}

Desde diciembre de 1991 a diciembre de 2012, 322 pacientes con IM tuvieron una reparación valvular mitral (RpVM), de acuerdo a los principios y técnicas de Carpentier, con control ecocardiográfico transesofágico intraoperatorio. Ciento noventa y ocho pacientes $(61,5 \%)$ fueron hombres. Estos predominaron en todas las etiologías, salvo la reumática en que las mujeres fueron mayoría (Tabla 1). La edad promedio fue 60 años (IC 95\%; 58,2-61,5), con un margen de 18 a 89 años. No hubo diferencias de edad entre ambos sexos $(\mathrm{p}=0,49)$, pero sí según la etiología de la IM (Tabla 1). La IM preoperatoria fue severa en $79 \%$ de los casos, moderada en $19 \%$ y leve en $2 \%$ restante. La Tabla 2 detalla el grado de IM preoperatoria según la etología de esta. La capacidad funcional (CF) se consignó en 197 pacientes (61\% de los casos), de los cuales 41 (21\%) estaban en CF I-II y 156 (79\%) en CF III-IV.

Durante el período en estudio, otros 563 pacientes tuvieron un recambio valvular mitral por IM. Así, del total de 885 pacientes operados por IM durante este período, 36\% tuvo una RpVM.

\section{Método}

Para la identificación de los pacientes se revisó la base de datos de nuestro Servicio de Cirugía Cardiovascular. Identificados los pacientes, se revisaron los protocolos operatorios y los informes angiográficos y ecocardiográficos disponibles. La supervivencia se certificó a través del Registro Civil e Identificación de Chile.

\section{Técnica quirúrgica}

Todos los pacientes fueron operados con circulación extracorpórea con hipotermia sistémica

Tabla 2. Magnitud de la insuficiencia mitral pre operatoria según etiología de la insuficiencia mitral

\begin{tabular}{|lccc|}
\hline $\begin{array}{l}\text { Etiología } \\
\text { valvular }\end{array}$ & $\begin{array}{c}\text { Leve } \\
\mathbf{n}(\%)\end{array}$ & $\begin{array}{c}\text { Moderada } \\
\mathbf{n}(\%)\end{array}$ & $\begin{array}{l}\text { Severa } \\
\mathbf{n}(\%)\end{array}$ \\
Degenerativa & $0(0)$ & $7(5)$ & $137(95)$ \\
Isquémica & $2(2)$ & $33(32)$ & $69(64)$ \\
Infecciosa & $4(11)$ & $8(22)$ & $24(67)$ \\
Reumática & $1(6)$ & $6(33)$ & $11(61)$ \\
Otras & $0(0)$ & $7(35)$ & $13(65)$ \\
Total & $7(2)$ & $61(19)$ & $254(79)$ \\
\hline
\end{tabular}


entre $28^{\circ} \mathrm{C}$ y $30^{\circ} \mathrm{C}$. La protección miocárdica se efectuó con solución cardioplégica cristaloide infundida a $4^{\circ} \mathrm{C}$ por vía anterógrada (raíz aórtica) y retrógrada (seno coronario) ${ }^{15}$.

En la mayoría de los casos se utilizó un acceso auricular izquierdo transeptal y superior combinado ${ }^{16}$.

\section{Análisis estadístico}

Las variables categóricas se expresan en porcentaje y las variables numéricas en promedio e Intervalo de Confianza 95\% (IC95\%) o mediana y margen, según su distribución. Las diferencias entre variables numéricas fueron establecidas por medio del test $t$-Student para variables con distribución normal demostrada por el método de Kolmogorov-Smirnov. Aquellas que no se distribuyeron normalmente fueron comparadas mediante el test de rangos de Wilcoxson. Las curvas de supervivencia se construyeron por medio del método de Kaplan-Meier. Un valor alfa de 5\% fue definido a priori para determinar diferencias estadísticas. Este análisis estadístico fue realizado por medio del paquete estadístico SPSS en su versión 17.0 (SPSS Inc., Chicago, USA).

\section{Resultados}

\section{Etiología y lesión valvular}

La etiología de la IM fue degenerativa en 144 pacientes (45\%), isquémica en $104(32 \%)$, infecciosa en $36(11 \%)$, reumática en $18(5 \%)$ y otras en $20(6 \%)$.

Las lesiones valvulares más frecuentes fueron la dilatación del anillo mitral en 50,6\% de los casos, la rotura de cuerdas tendíneas del velo posterior en $26,4 \%$ y el alargamiento de cuerdas tendíneas en $21 \%$ de los casos (Tabla 3 ).

\section{Enfermedad coronaria}

Se efectuó una coronariografía pre-operatoria en $96 \%$ de los pacientes con IM isquémica y en $56 \%$ de las no isquémicas. Se encontraron lesiones coronarias en $95 \%$ y $24 \%$ de los casos, respectivamente. La Tabla 4 detalla lo anterior según etiología específica. De los 4 casos con IM isquémica en los que no se hizo coronariografía pre $\mathrm{RpVM}, 3$ correspondieron a infartos antiguos que desarrollaron posteriormente IM y no se estimó pertinente repetir la coronariografía, y el cuarto a un paciente con cirugía coronaria reciente que en el postoperatorio presentó una IM.

\section{Valvulopatía asociada}

Noventa y siete pacientes (30\%) presentaron alguna valvulopatía asociada. De estos, 50 casos $(52 \%)$ correspondieron a valvulopatía aórtica,

Tabla 3. Hallazgos anátomo patológicos intraoperatorios encontrados en la cirugía reparadora de la insuficiencia mitral

\begin{tabular}{|lrc|}
\hline & n & $\begin{array}{c}\text { Casos } \\
\%\end{array}$ \\
\hline Dilatación del anillo & 163 & 50,6 \\
\hline Alargamiento cuerdas tendíneas & 68 & 21,1 \\
\hline Rotura de cuerdas posteriores & 85 & 26,4 \\
\hline Rotura cuerdas anteriores & 29 & 9,0 \\
\hline Perforación del velo anterior & 14 & 4,3 \\
Perforación del velo posterior & 8 & 2,5 \\
Tracción del velo posterior & 45 & 14,0 \\
Retracción-fibrosis del velo & 24 & 7,5 \\
Fusión comisural & 13 & 4,0 \\
Otras & 25 & 7,8 \\
\hline
\end{tabular}

Tabla 4. Coronariografía pre operatoria y lesiones coronarias según etiología de la insuficiencia mitral

\begin{tabular}{|c|c|c|c|c|c|c|}
\hline Características & $\begin{array}{c}\text { Degenerativa } \\
\text { n (\%) }\end{array}$ & $\begin{array}{c}\text { Isquémica } \\
\text { n (\%) }\end{array}$ & $\begin{array}{c}\text { Infecciosa } \\
\text { n (\%) }\end{array}$ & $\begin{array}{c}\text { Reumática } \\
\text { n (\%) }\end{array}$ & $\begin{array}{l}\text { Otras } \\
\text { n (\%) }\end{array}$ & $\begin{array}{l}\text { Total } \\
\text { n }(\%)\end{array}$ \\
\hline Coronariografía pre operatoria & $100(69,4)$ & $100(96,2)$ & $7(19,4)$ & $5(27,8)$ & $11(55,0)$ & $223(69,3)$ \\
\hline \multicolumn{7}{|l|}{ Enfermedad coronaria } \\
\hline Sin lesión & $73(73,0)$ & $4(4,0)$ & $6(85,7)$ & $4(80,0)$ & $11(100)$ & $98(44,0)$ \\
\hline Alguna lesión & $27(27,0)$ & $96(96,0)$ & $1(14,3)$ & $1(20,0)$ & $0 \quad(0,0)$ & $125(56,0)$ \\
\hline 1 vaso & $12(12,0)$ & $8(8,0)$ & $0 \quad(0)$ & $1(20,0)$ & $0 \quad(0,0)$ & $21 \quad(9,7)$ \\
\hline 2 vasos & $7 \quad(7,0)$ & $14(14,0)$ & $1(14,3)$ & $0 \quad(0)$ & $0 \quad(0,0)$ & $22 \quad(9,7)$ \\
\hline 3 o más vasos & $8(8,0)$ & $74(74,0)$ & $0 \quad(0)$ & $0 \quad(0,0)$ & $0 \quad(0,0)$ & $82(36,6)$ \\
\hline
\end{tabular}


Tabla 5. Técnicas quirúrgicas utilizadas en la cirugía de reparación mitral según etiología de insuficiencia mitral

\begin{tabular}{|c|c|c|c|c|c|c|c|}
\hline Tipo de reparación mitral & $\begin{array}{c}\text { Degenerativa } \\
\text { n }(\%)\end{array}$ & $\begin{array}{l}\text { Isquémica } \\
\text { n }(\%)\end{array}$ & $\begin{array}{l}\text { Infecciosa } \\
\text { n (\%) }\end{array}$ & $\begin{array}{c}\text { Reumática } \\
\text { n (\%) }\end{array}$ & & $\begin{array}{l}\text { Otras } \\
\text { n (\%) }\end{array}$ & $\begin{array}{l}\text { Total } \\
\text { n }(\%)\end{array}$ \\
\hline Anuloplastía mitral & $133(92,4)$ & $104(100)$ & $32(88,9)$ & $17(94,4)$ & 18 & $(90)$ & $304(94,4)$ \\
\hline $\begin{array}{l}\text { Resección cuadrilateral del velo } \\
\text { posterior }\end{array}$ & $110(76,4)$ & $2(1,9)$ & $12(33,3)$ & $0 \quad(0,0)$ & 1 & $(5,0)$ & $125(38,8)$ \\
\hline Transferencia de cuerdas tendíneas & $18(12,5)$ & $1(1,0)$ & $3(8,3)$ & $0 \quad(0,0)$ & 0 & $(0,0)$ & $22(6,8)$ \\
\hline Acortamiento de cuerdas tendíneas & $4(2,8)$ & $0(0,0)$ & $0 \quad(0,0)$ & $0 \quad(0,0)$ & 0 & $(0,0)$ & $4(1,2)$ \\
\hline Resección de vegetaciones & $0 \quad(0,0)$ & $0(0,0)$ & $8(22,0)$ & $0 \quad(0,0)$ & 0 & $(0,0)$ & $8(2,5)$ \\
\hline Cierre de perforación (cleft) & $6(4,2)$ & $1(1,0)$ & $8(22,2)$ & $2(11,2)$ & & $(10,0)$ & $19(5,9)$ \\
\hline Cierre de indentación & $1(0,7)$ & $2(1,9)$ & $0 \quad(0,0)$ & $0 \quad(0,0)$ & 0 & $(0,0)$ & $3(0,9)$ \\
\hline Reparación comisural & $7(4,9)$ & $2(1,9)$ & $3(8,3)$ & $11(61,1)$ & 0 & $(0,0)$ & $23(7,1)$ \\
\hline Reconstrucción anular & $1(0,7)$ & $0(0,0)$ & $2(5,6)$ & $0 \quad(0,0)$ & 0 & $(0,0)$ & $3(0,9)$ \\
\hline $\begin{array}{l}\text { Papilotomía o sección de cuerdas } \\
\text { tendíneas fibrosadas }\end{array}$ & $0 \quad(0,0)$ & $5(4,8)$ & $0 \quad(0,0)$ & $7(38,8)$ & 1 & $(5,0)$ & $13(4,0)$ \\
\hline Resuturación de cuerdas tendíneas & $0 \quad(0,0)$ & $0(0,0)$ & $2(5,6)$ & $0 \quad(0,0)$ & 0 & $(0,0)$ & $2(0,6)$ \\
\hline
\end{tabular}

$36(37 \%)$ a insuficiencia tricuspídea y en 10 casos (10\%) ambas válvulas estaban comprometidas. En 22 pacientes las lesiones valvulares asociadas eran leves, por lo que no se efectuó ningún procedimiento quirúrgico sobre estas.

\section{Técnica quirúrgica}

La Tabla 5 detalla las técnicas quirúrgicas utilizadas, según etiología. La más utilizada fue la resección cuadrilateral del velo posterior $(39 \%$ de los casos). Se utilizó un anillo protésico en $100 \%$ de los casos de IM isquémica y en $92 \%$ de las no isquémicas. La Figura 1 detalla los tipos de anillos utilizados. El anillo Carpentier-McCarthy-Adams sólo se utilizó en la IM isquémica. La mediana del tamaño de anillo fue 30, con un margen entre 26 y 38. Para la IM isquémica la mediana fue 28 (26 a 34) y para la IM degenerativa 32 (26 a 38) $(\mathrm{p}<0,001)$. La orejuela izquierda se cerró en 79 casos $(25 \%)$.

\section{Revascularización miocárdica}

En 125 pacientes (39\%) se efectuó una revascularización miocárdica, con un promedio de 2,7 puentes/paciente. Esta se efectuó en $92 \%$ de los casos de IM isquémica y en $13 \%$ de las no isquémicas. La arteria mamaria interna izquierda se utilizó en $45,6 \%$ de todos los casos revascularizados.

\section{Procedimiento valvular asociado}

En 75 pacientes (23\%) se efectuaron un total de 83 procedimientos valvulares asociados. De estos, 18 pacientes $(24 \%)$ tenían una IM isquémica y 57 (76\%) una IM no isquémica. Los procedimientos más frecuentes fueron el reemplazo valvular aórtico y la anuloplastía tricuspídea. La Tabla 6 muestra los procedimientos valvulares asociados según etiología específica.

\section{Mortalidad operatoria}

La mortalidad hospitalaria o hasta 30 días de la operación fue $7,5 \%$ (24 pacientes). Para la IM isquémica fue $13 \%$ y para la no isquémica $4,4 \%$ $(\mathrm{p}<0,005)$. La mortalidad para la IM degenerativa aislada electiva fue $0,7 \%$ (1 paciente).

\section{Supervivencia}

El seguimiento alejado se completó en 100\% de los casos. La supervivencia global promedio fue14,1 años (IC 95\%; 13-15,2), siendo 9,3 años (IC 95\%; 7,8-10,9) para la IM isquémica y 16 años (IC 95\%; 14,7-17,2) para la IM no isquémica, con un Hazard Ratio de 2,7 (IC 95\%; 1,8-3,9) $(\mathrm{p}<0,001)$. La supervivencia promedio para la IM degenerativa fue 16,6 años (IC 95\%; 15-18,2). La supervivencia global a 5, 10, 15 y 20 años fue $79 \%$, $63 \%$, 54\% y $42 \%$, respectivamente (Figura 2). La supervivencia a 5 y 10 años para la IM isquémica 


\begin{tabular}{|c|c|c|}
\hline Classic & & $\begin{array}{c}136 \text { casos } \\
(45 \%)\end{array}$ \\
\hline Physio-Ring & & $\begin{array}{c}117 \text { casos } \\
(39 \%)\end{array}$ \\
\hline Mc'Carthy-Adams & $S^{2}$ & $\begin{array}{c}31 \text { casos } \\
(10 \%)\end{array}$ \\
\hline Physio-Ring II & & $\begin{array}{c}13 \text { casos } \\
(4 \%)\end{array}$ \\
\hline Myxo Etlogix & & $\begin{array}{c}5 \text { casos } \\
(2 \%)\end{array}$ \\
\hline
\end{tabular}

Figura 1. Anillos protésicos Carpentier-Edwards utilizados según su frecuencia.

Tabla 6. Procedimientos valvulares asociados según etiología de la insuficiencia mitral

\begin{tabular}{|lccccccc|}
\hline $\begin{array}{l}\text { Procedimiento valvular } \\
\text { asociado }\end{array}$ & $\begin{array}{c}\text { Degenerativa } \\
\mathbf{n}(\%)\end{array}$ & $\begin{array}{c}\text { Isquémica } \\
\mathbf{n}(\%)\end{array}$ & $\begin{array}{c}\text { Infecciosa } \\
\mathbf{n}(\%)\end{array}$ & $\begin{array}{c}\text { Reumática } \\
\mathbf{n}(\%)\end{array}$ & $\begin{array}{c}\text { Otras } \\
\mathbf{n}(\%)\end{array}$ & $\begin{array}{c}\text { Total } \\
\mathbf{n}(\%)\end{array}$ \\
Recambio aórtico & $8(5,6)$ & $5(4,8)$ & $12(25,0)$ & $1(5,6)$ & $12(60,0)$ & $38(11,8)$ \\
Reparación aórtica & $2(1,4)$ & $0(0,0)$ & $6(12,5)$ & $1(5,6)$ & 0 & $(0,0)$ & $9(2,8)$ \\
Anuloplastía tricuspídea & $9(6,2)$ & $14(13,5)$ & $4(8,3)$ & $3(16,6)$ & $5(25)$ & $35(10,9)$ \\
Reparación pulmonar & $0(0,0)$ & $0(0,0)$ & $0(0,0)$ & $0(0,0)$ & 1 & $(5,0)$ & $1(0,3)$ \\
Total & $19(13,2)$ & $19(18,3)$ & $22(45,8)$ & $5(27,8)$ & $18(90,0)$ & $83(25,8)$ \\
\hline
\end{tabular}

fue $64 \%$ y $44 \%$ y para la no isquémica de $85 \%$ y $72 \%$, respectivamente. La supervivencia para la IM degenerativa fue $90 \%$ y $76 \%$, a 5 y 10 años, respectivamente $(\mathrm{p}<0,001)$ (Figura 3 ). No se observaron diferencias en cuanto a mortalidad alejada según el sexo $(\mathrm{p}=0,306)$.
Durante el seguimiento fallecieron 117 pacientes. De estos, 70 (60\%) fallecieron por causa cardiaca y $47(40 \%)$ por causa no cardiaca. Así, la supervivencia libre de muerte cardiaca a 5 y 10 años fue de $84 \%$ y $77 \%$, respectivamente (Figura 4). En cuanto a la etiología y causa de mortali- 


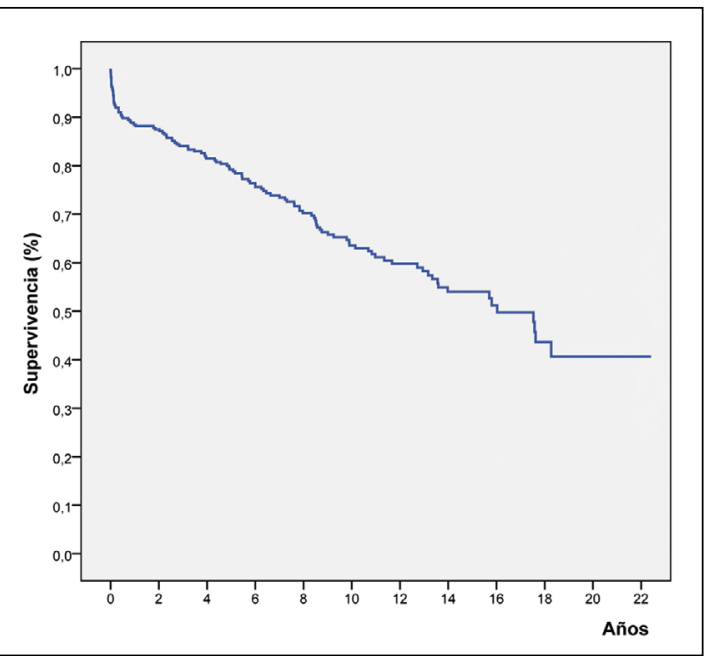

Figura 2. Análisis de supervivencia global alejada posterior a cirugía de reparación mitral por insuficiencia mitral estimada por el método de Kaplan-Meier.

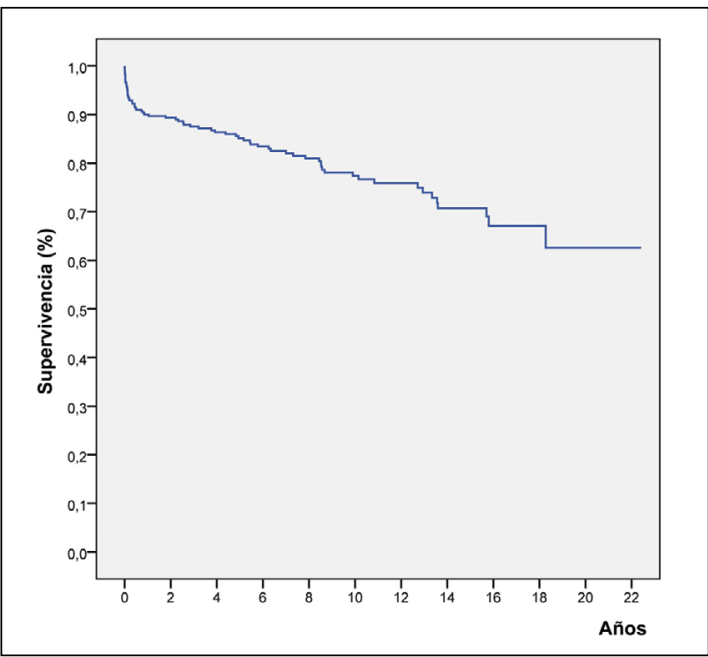

Figura 4. Análisis de supervivencia libre de muerte cardiaca posterior a cirugía de reparación mitral por insuficiencia mitral estimada por el método de Kaplan-Meier.

dad alejada, de los 54 pacientes con IM isquémica fallecidos, $38(70 \%)$ fallecieron por causa cardiaca y $16(30 \%)$ por causa no cardiaca. De los 63 pacientes fallecidos con IM no isquémica, 32 (51\%) murieron por causa cardiaca y 31 (49\%) por causa no cardiaca. La Figura 5 muestra la supervivencia libre de muerte cardiaca para la IM isquémica y no isquémica.

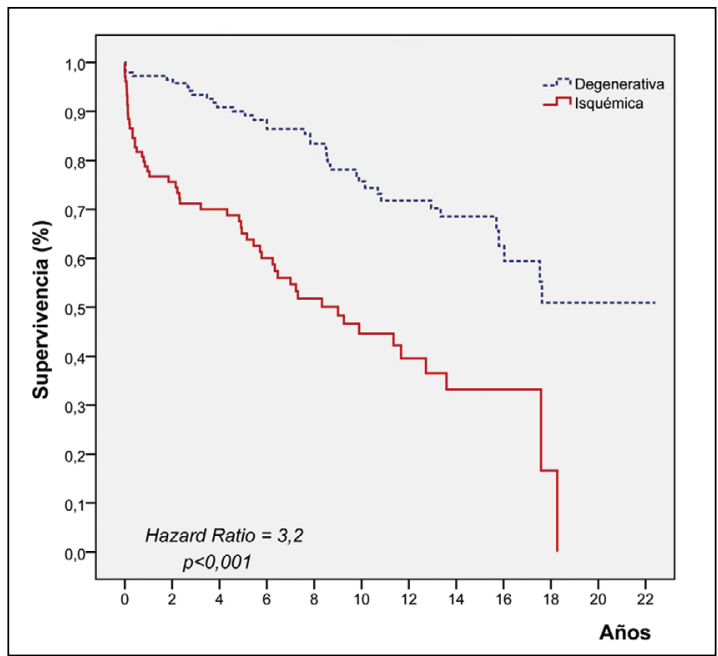

Figura 3. Análisis de supervivencia alejada posterior a cirugía de reparación mitral según etiología de insuficiencia mitral estimado por el método de Kaplan-Meier.

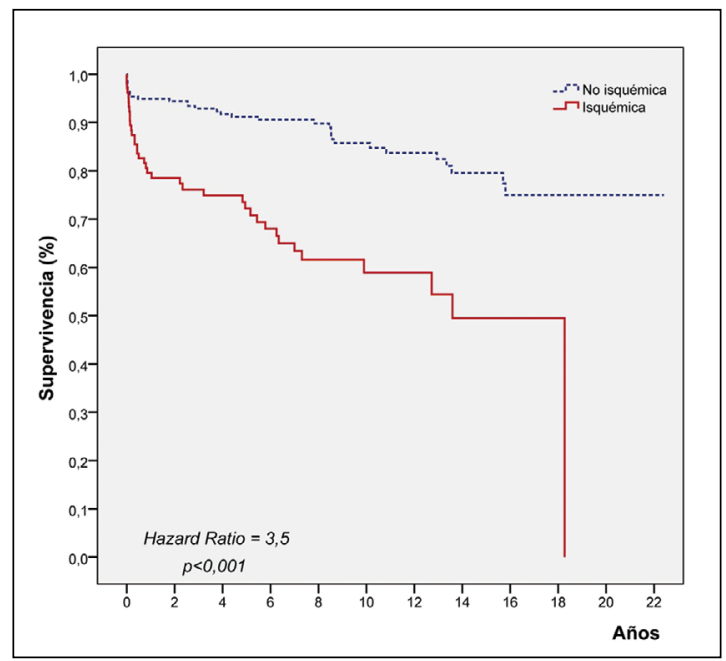

Figura 5. Análisis de supervivencia libre de muerte cardiaca posterior a cirugía de reparación mitral por insuficiencia mitral según etiología isquémica estimada por el método de Kaplan-Meier.

\section{Variables asociadas a mortalidad}

Se realizó un análisis multivariado para identificar las variables asociadas a mortalidad (Tabla 7). De acuerdo a éste, edad (OR 1,06; IC $95 \% ; 1,04-1,08$ ), valvulopatía asociada (OR 2,0; IC $95 \% ; 1,1-3,5)$ y etiología isquémica (OR 1,8; IC $95 \% ; 1,1-3,1)$ fueron identificadas como las principales variables asociadas a mortalidad ajustadas 
por sexo. Por el contrario, el cierre de la orejuela izquierda se identificó como un factor protector de mortalidad (OR 0,25; IC 95\%; 0,13-0,5).

\section{Seguimiento ecocardiográfico}

Se completó en $69 \%$ de los casos (223 pacientes), con un promedio de 54 meses (IC 95\%; 46,4-62). La Tabla 8 resume los hallazgos ecocardiográficos en cuanto a magnitud de la IM y etiología. La gran mayoría (82\%) de los pacientes no tenía IM o esta era leve y no se encontraron diferencias significativas según la etiología de la $\operatorname{IM}(\mathrm{p}=0,49)$.

\section{Reoperación}

Trece pacientes fueron reoperados de la VM en nuestra institución durante el seguimiento. Diez lo fueron por recidiva de la IM y otros 3 por endocarditis infecciosa. Otros 2 pacientes fueron reoperados en otras instituciones. Así, el tiempo promedio libre de reoperación mitral fue 21,6 años (IC 95\%; 21,1-22,2). La probabilidad de estar libre de reoperación a 20 años fue $92 \%$ (Figura 6). No hubo diferencia significativa en cuanto a reoperación valvular mitral entre la IM isquémica y la IM degenerativa (Figura 7). En todos los casos, la VM fue reemplazada.

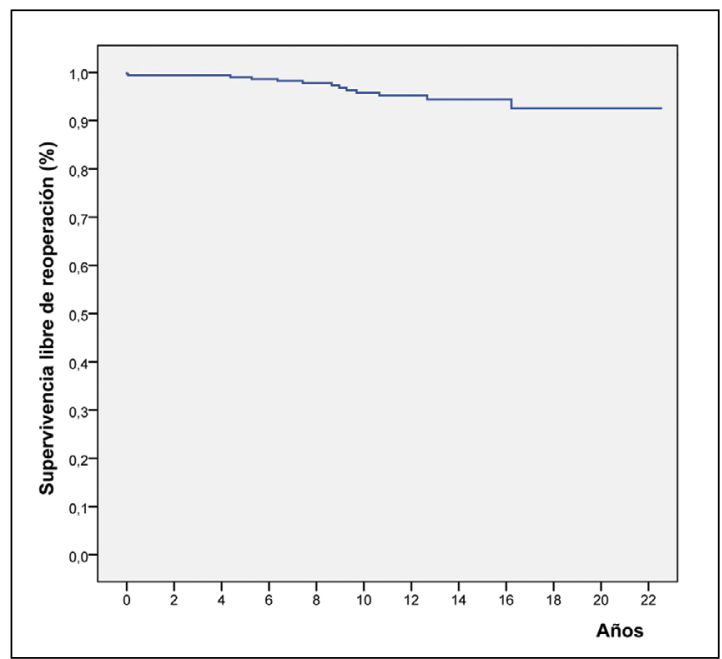

Figura 6. Análisis de supervivencia libre de reoperación mitral posterior a cirugía de reparación mitral por insuficiencia mitral estimada por el método de Kaplan-Meier.
Tabla 7. Regresión logística de principales variables asociadas a mortalidad alejada en cirugía de reparación mitral

\begin{tabular}{|lccccc|}
\hline Variable & B & Wald & OR & IC 95\% & p \\
\hline Edad & 0,06 & 26,9 & 1,06 & $1,04-1,08$ & $<0,001$ \\
$\begin{array}{l}\text { Etiología } \\
\text { isquémica }\end{array}$ & 0,6 & 4,7 & 1,82 & $1,06-3,12$ & 0,03 \\
$\begin{array}{l}\text { Valvulopatía } \\
\text { asociada }\end{array}$ & 0,7 & 5,8 & 2,0 & $1,14-3,5$ & 0,16 \\
$\begin{array}{l}\text { Cierre de } \\
\text { orejuela }\end{array}$ & $-1,38$ & 15,4 & 0,25 & $0,13-0,5$ & $<0,001$ \\
\hline
\end{tabular}

Tabla 8. Magnitud de la insuficiencia mitral post operatoria alejada según etiología de la insuficiencia mitral en paciente con seguimiento ecocardiográfico posterior al alta

\begin{tabular}{|lccccc|}
\hline $\begin{array}{l}\text { Etiología } \\
\text { valvular }\end{array}$ & $\begin{array}{c}\text { Mínima o } \\
\text { ausente } \\
\mathbf{n}(\%)\end{array}$ & $\mathbf{n}(\%)$ & $\mathbf{n}(\%)$ & $\mathbf{n ~ ( \% )}$ \\
Degenerativa & $55(52,4)$ & $34(32,4)$ & $14(13,3)$ & $2(1,9)$ \\
Isquémica & $36(52,9)$ & $19(27,9)$ & $8(11,8)$ & $5(7,4)$ \\
Infecciosa & $14(56,0)$ & $7(28,0)$ & $2(8,0)$ & $2(8,0)$ \\
Reumática & $5(35,7)$ & $4(28,6)$ & $5(35,7)$ & $0(0,0)$ \\
Otras & $7(63,6)$ & $1(9,1)$ & $3(27,3)$ & $0(0,0)$ \\
Total & $117(52,5)$ & $65(29,2)$ & $32(14,3)$ & $9(4,0)$ \\
\hline
\end{tabular}

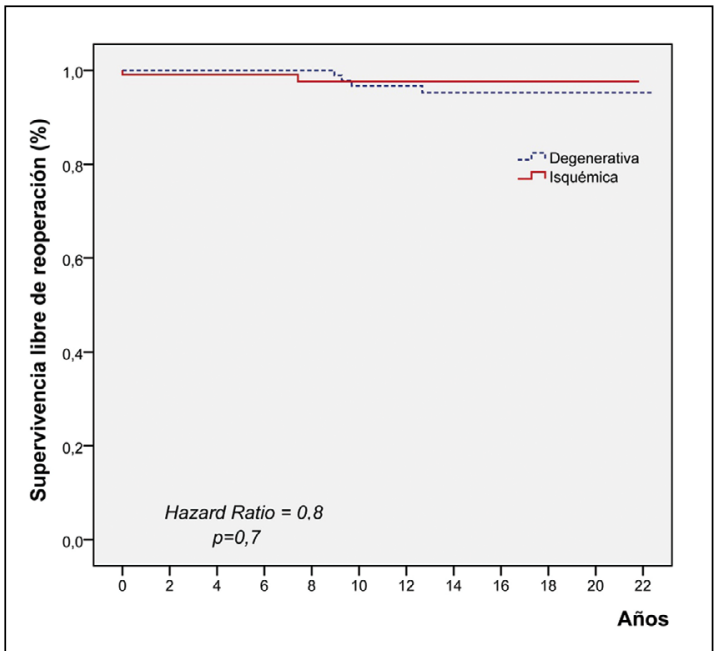

Figura 7. Análisis de supervivencia libre de reoperación mitral posterior a cirugía de reparación mitral por insuficiencia mitral según etiología estimada por el método de Kaplan-Meier. 


\section{Discusión}

En la década de 1970-79, Carpentier introdujo el concepto de "análisis valvular funcional", para entender el mecanismo y las causas de la IM, a partir del cual creó la anuloplastía mitral "remodeladora”, en contraposición a la anuloplastía "reductora", la que devuelve al anillo mitral dilatado su forma de "riñón" que tiene en sístole, mediante un anillo protésico así configurado. Pero, como raramente la dilatación del anillo es la única causa de la IM, y por el contrario, con frecuencia existen lesiones de los velos y del aparato sub-valvular, Carpentier desarrolló sucesivamente técnicas quirúrgicas complementarias para tratar estas lesiones $^{1-3}$.

Inicialmente, los pacientes más beneficiados de la RpVM fueron aquellos con secuelas reumáticas. En la década de1980-89 se observó un aumento de la enfermedad degenerativa y fueron estos pacientes en los que los procedimientos reconstructores tuvieron su mayor aplicación. En la década de 1990-99, los beneficios de la RpVM se establecieron sólidamente para estos, por lo que la atención se dirigió ahora a la IM isquémica, desarrollándose conceptos y procedimientos quirúrgicos específicos para esta ${ }^{14,19-22}$.

Actualmente, la IM degenerativa y la IM isquémica constituyen los 2 grandes grupos de pacientes en los que la RpVM tiene su mayor aplicación. Existen sí, diferencias importantes entre ambos. Así, los pacientes con IM isquémica tienden a ser mayores, en nuestra experiencia, en promedio 6 años más que los pacientes con IM degenerativa. Obviamente, los primeros van a requerir una revascularización miocárdica concomitante con la RpVM y los segundos tienen una mayor incidencia de procedimientos valvulares asociados. Pero, la diferencia fundamental es que la IM isquémica es una enfermedad ventricular (la VM es esencialmente normal) y la IM degenerativa es una enfermedad valvular propiamente tal. Lo anterior tiene claras consecuencias en las técnicas quirúrgicas a utilizar y en el pronóstico de los pacientes, lo que queda de manifiesto en nuestra experiencia ${ }^{14,19-23}$.

En cuanto a la técnica quirúrgica, en la IM isquémica la operación es la anuloplastía mitral, la que persigue aumentar la coaptación de los velos mitrales, a través de una sobre-reducción del diámetro antero-posterior del anillo mitral ${ }^{20-22}$. Es por esto que la mediana de anillo utilizado en esta experiencia fue 28 , en contraposición a la mediana de la serie global, que fue 30 y de la IM degenerativa, que fue 32 . El anillo CarpentierMcCarthy-Adams, especialmente diseñado para la IM isquémica, incorpora ya una sobre-reducción del diámetro antero-posterior ${ }^{24}$. En cualquier caso, el anillo protésico debe ser un anillo completo ${ }^{25,26}$. Los procedimientos limitados al anillo mitral posterior se acompañan de una alta recidiva de IM, lo que obviamente repercute en la incidencia de reoperación y en la supervivencia alejada ${ }^{20-22,24-26}$.

Desde el inicio de nuestra experiencia hemos respetado estos conceptos, lo que explicaría la baja incidencia de recidiva de IM y de reoperación en nuestros pacientes con IM isquémica.

Por el contrario, en la IM degenerativa, la anuloplastía mitral es un complemento de la $\mathrm{RpVM}$. La continencia valvular debe conseguirse con los procedimientos efectuados sobre los otros componentes del aparato valvular, como los velos o cuerdas tendíneas, a través de resecciones cuadrilaterales, transferencias o acortamientos de cuerdas, reconstrucción comisural, etc., como lo ha sido en esta serie, lo que se ha traducido, nuevamente, en una baja incidencia de reoperación y de recidiva de $\mathrm{IM}^{1-3,10,11,13,27}$. En relación a este último aspecto, es necesario enfatizar que en casi un tercio de los pacientes no se dispuso de un ecocardiograma alejado.

El estricto apego a estos conceptos permite también explicar la baja mortalidad operatoria de la serie global y de la IM degenerativa en particular. Si bien la mortalidad de la IM isquémica fue $13 \%$, está dentro de la reportada. Igualmente, permite explicar la excelente supervivencia alejada de la serie en general y de la IM degenerativa, en particular, la que para esta última fue de $90 \%$ y $76 \%$, a 5 y 10 años. La marcada menor supervivencia de los pacientes con IM isquémica ( $64 \%$ y $44 \%$, respectivamente) está dada por la disfunción ventricular más que por la IM, como se puede deducir del hecho de que en esta experiencia $70 \%$ de estos pacientes falleció por causa cardiaca ${ }^{14,19,28-30}$.

Otros factores que permiten explicar estos buenos resultados son el control ecocardiográfico transesofágico intraoperatorio, la protección miocárdica con solución cardioplégica infundida por vía retrógrada y el acceso auricular izquierdo transeptal y superior combinado, utilizados en nuestros pacientes desde el inicio de nuestra experiencia ${ }^{9,15,16}$. Así, en ningún caso se permitió 
una IM residual mayor que leve en el control ecocardiográfico intraoperatorio. Publicaciones recientes han demostrado que una IM intraoperatoria mayor a leve se acompaña de una mayor probabilidad de desarrollar IM severa en el seguimiento ${ }^{31}$. Por otra parte, el uso de solución cardioplégica por vía retrógrada no sólo permite una mejor protección miocárdica, sino que, además, no interfiere con la exposición de la válvula mitral, lo que es fundamental para efectuar una RpVM. Por lo mismo, en prácticamente todos nuestros pacientes utilizamos un acceso auricular izquierdo transeptal y superior combinado, el que permite una excelente exposición de la VM.

Finalmente, si bien era esperable que los principales factores asociados a mortalidad fueran la edad, la valvulopatía asociada y la etiología isquémica, que el cierre de la orejuela izquierda sea un factor protector de mortalidad fue una sorpresa, lo que someteremos a un mayor análisis a futuro, si bien es posible especular, como explicación, una menor incidencia de eventos tromboembólicos.

\section{Referencias}

1. Carpentier A, Deloche A, Dauptain J, Soyer R, Blondeau $\mathrm{P}$, Piwnica A, et al. A new reconstructive operation for correction of mitral and tricuspid insufficiency. J Thorac Cardiovasc Surg 1971; 61: 1-13.

2. Carpentier A, Relland J, Deloche A, Fabiani J, D'allaines C, Blondeau $\mathrm{P}$, et al. Conservative management of the prolapsed mitral valve. Ann Thorac Surg 1978; 26: 294302.

3. Carpentier A. Cardiac valve surgery-The "French correction". J Thorac Cardiovasc Surg 1983; 86: 323-37.

4. Cosgrove MD, Chávez AM, Lytle BW, Gill CC, Stewart RW, Taylor PC, et al. Result of mitral valve reconstruction. Circulation 1986; 74 (suppl): I 82-7.

5. Loop FD. Long-term results of mitral valve repair. Semin Thorac Cardiovasc Surg 1989; 1: 203-10.

6. Galloway AC, Colvin SB, Baumann G, Esposito R, Vohra $\mathrm{R}$, Harty $\mathrm{S}$, et al. Long-term results of mitral valve reconstruction with Carpentier techniques in 148 patients with mitral insufficiency. Circulation 1988; 78 (suppl): I 97-105.

7. Galloway AC, Colvin SB, Baumann FG, Grossi EA, Ribakove GH, Harty S, et al. A comparison of mitral valve reconstruction with mitral valve replacement: intermediate-term results. Ann Thorac Surg 1989; 47: 655-62.
8. Zalaquett R. Cirugía reparadora de la insuficiencia valvular mitral. Rev Chil Cir 1994; 46: 127-36.

9. Zalaquett R, Moran S, Irarrázaval MJ, Maturana G, Braun S, Chamorro G, et al. Reconstrucción válvula mitral. Cirugía reparadora de la insuficiencia valvular mitral con evaluación ecocardiográfica transesofágica intraoperatoria. Rev Chil Cardiol 1996; 15: 78-84.

10. Zalaquett R, Chamorro S, Braun S, Garrido L, Howard M, Moran S, et al. Resultados alejados de la cirugía reconstructora de la insuficiencia mitral. Rev Med Chile 1999; 127: 1093-100.

11. Zalaquett R, Campla C, Córdova S, Braun S, Chamorro G, Irarrázaval M, et al. Resultados alejados de la cirugía reparadora de la insuficiencia mitral degenerativa. Rev Med Chile 2003; 131: 1355-64.

12. Zalaquett R, Garrido L, Casas F, Moran S, Irarrázaval MJ, Becker $\mathrm{P}$, et al. Cirugía valvular reparadora en endocarditis infecciosa. Rev Med Chile 2004; 132: 307-15.

13. Zalaquett R, Scheu M, Campla C, Moran S, Irarrázaval $\mathrm{MJ}$, Becker P, et al. Resultados alejados de la reparación versus reemplazo en insuficiencia mitral degenerativa. Rev Med Chile 2005; 133: 1139-46.

14. Zalaquett R, Flores E, Cartajena F, Bachler S, Córdova S, Becker P, et al. Resultados alejados de la cirugía reparadora de la insuficiencia mitral isquémica. Rev Med Chile 2011; 139: 1544-52.

15. Zalaquett R, Becker P, Irarrázaval MJ, Moran S, Maturana G, Navarro M, et al. Cardioplegia retrógrada por canulación transauricular derecha del seno coronario. Rev Chil Cardiol 1993; 12: 68-71.

16. Zalaquett R, Moran S, Irarrázaval MJ. Maturana G, Navarro M, Braun S, et al. Acceso auricular izquierdo transeptal y superior combinado. Rev Chil Cir 1993; 45: 225-8.

17. Otto CM. Evaluation and management of chronic mitral regurgitation. N Engl J Med 2001; 345: 740-6.

18. Lillehei C, Levy M, Bonnabeau R. Mitral valve replacement with preservation of papillary muscles and chordate tendinae. J Thorac Cardiovasc Surg 1964; 47: 532-43.

19. Levine RA, Schammenthal E. Ischemic mitral regurgitation on the threshold of a solution. Circulation 2005; 112: 745-58.

20. Miller DC. Ischemic mitral regurgitation redux-to repair or to relace? J Thorac Cardiovasc Surg 2001; 122: 1059 62.

21. Bolling SF, Deeb GM, Bach DS. Mitral valve reconstruction in elderly, ischemic patients. Chest 1996; 109: 35-40.

22. Bolling SF, Pagani FD, Deeb GM, Bach DS. Intermediate term outcome of mitral reconstruction in cardiomyopathy. J Thorac Cardiovasc Surg 1998; 115: 381-6. 
23. Lung B. Management of ischaemic mitral regurgitation. Heart 2003; 89: 459-64.

24. Daimon M, Fakuda S, Adams DH, Mccarthy PM, Gillinov AM, Carpentier A, et al. Mitral valve repair with Carpentier-McCarthy-Adams IMR ETlogixannuloplasty ring for ischemic mitral regurgitation. Circulation 2006; 114: I-588-93.

25. Williams ML, Daneshmand MA, Jollis JG, Horton JR, Shaw LK, Swaminathan M, et al. Mitral gradients and frequency of recurrence of mitral regurgitation after ring annuloplasty for ischemic mitral regurgitation. Ann Thorac Surg 2009; 88: 1197-201.

26. Braun J, Van De Veire NR, Klautz RJ, Versteegh MI, Holman ER, Westenberg JJ, et al. Restrictive mitral annuloplasty cures ischemic mitral regurgitation and heart failure. Ann Thorac Surg 2008: 85: 430-6.

27. Carpentier AF, Lessana A, Relland JY, Belli E, Mihaileanu S, Barrebi AJ, et al. The "Physio-Ring": An advanced concept in mitral valve annuloplasty. Ann Thorac Surg 1995; 60: 1177-85.

28. DI Donato M, Frigiola A, Menicanti L, Boghdabi A, Badia T, Neagu A, et al. Moderate ischemic mitral regurgitation and coronary artery bypass surgery: Effect of mitral repair on clinical outcome. J Heart Valve Dis 2003; 12: 272-9.

29. Grossi EA, Bizekis CS, Lapietra A, Deriveaux CC, Galloway AS, Ribakove GH, et al. Late results of isolated mitral annuloplasty for "functional" ischemic mitral insufficiency. J Card Surg 2001; 16: 328-32.

30. Zalaquett R, Garrido C, Moran S, Irarrázaval MJ, Becker $\mathrm{P}$, Maturana G, et al. Cirugía de la insuficiencia mitral isquémica. Rev Med Chile 2002; 130: 9-16.

31. Kasegawa H, Shimokawa T, Shibazaki I, Hayashi H, Koyanagi T, Ida T. Mitral valve repair for anterior leaflet prolapse with expanded polytetrafluoroethylene sutures. Ann Thorac Surg 2006; 81: 1625-31. 